\title{
Performance evaluation of space-time-frequency spreading for MIMO OFDM-CDMA systems
}

\author{
Haysam Dahman* and Yousef Shayan
}

\begin{abstract}
In this article, we propose a multiple-input-multiple-output, orthogonal frequency division multiplexing, codedivision multiple-access (MIMO OFDM-CDMA) scheme. The main objective is to provide extra flexibility in user multiplexing and data rate adaptation, that offer higher system throughput and better diversity gains. This is done by spreading on all the signal domains; i.e, space-time frequency spreading is employed to transmit users' signals. The flexibility to spread on all three domains allows us to independently spread users' data, to maintain increased system throughput and to have higher diversity gains. We derive new accurate approximations for the probability of symbol error and signal-to-interference noise ratio (SINR) for zero forcing (ZF) receiver. This study and simulation results show that MIMO OFDM-CDMA is capable of achieving diversity gains significantly larger than that of the conventional 2-D CDMA OFDM and MIMO MC CDMA schemes.
\end{abstract}

Keywords: code-division multiple-access (CDMA), diversity, space-time-frequency spreading, multiple-input multiple-output (MIMO) systems, orthogonal frequency-division multiplexing (OFDM), 4th generation (4G)

\section{Introduction}

Modern broadband wireless systems must support multimedia services of a wide range of data rates with reasonable complexity, flexible multi-rate adaptation, and efficient multi-user multiplexing and detection. Broadband access has been evolving through the years, starting from 3G and High-Speed Downlink Packet Access (HSDPA) to Evolved High Speed Packet Access (HSPA +) [1] and Long Term Evolution (LTE). These are examples of next generation systems that provide higher performance data transmission, and improve end-user experience for web access, file download/upload, voice over IP and streaming services. HSPA+ and LTE are based on shared-channel transmission, so the key features for an efficient communication system are to maximize throughput, improve coverage, decrease latency and enhance user experience by sharing channel resources between users, providing flexible link adaptation, better coverage, increased throughput and easy multi-user multiplexing.

An efficient technique to be used in next generation wireless systems is OFDM-CDMA. OFDM is the main

\footnotetext{
* Correspondence: h_dahman@ece.concordia.ca

Department of Electrical Engineering, Concordia University, Montreal, QC,
} Canada air interface for LTE system, and on the other hand, CDMA is the air interface for HSPA+, so by combining both we can implement a system that benefits from both interfaces and is backward compatible to 3G and 4G systems. Various OFDM-CDMA schemes have been proposed and can be mainly categorized into two groups according to code spreading direction [2-5]. One is to spread the original data stream in the frequency domain; and the other is to spread in the time domain.

The key issue in designing an efficient system is to combine the benefits of both spreading in time and frequency domains to develop a scheme that has the potential of maximizing the achievable diversity in a multi-rate, multiple-access environment. In [6], it has been proposed a novel joint time-frequency 2-dimensional (2D) spreading method for OFDM-CDMA systems, which can offer not only time diversity, but also frequency diversity at the receiver efficiently. Each user will be allocated with one orthogonal code and spread its information data over the frequency and time domain uniformly. In this study, it was not mentioned how this approach will perform in a MIMO environment, specially in a downlink transmission. On the other hand, in [7], it was proposed a technique, called space-time spreading (STS), that improves the downlink 
performance, however they do not consider the multiuser interference problem at all. It was assumed that orthogonality between users can somehow be achieved, but in this article, this is a condition that is not trivially realized. Also, in [8], multicarrier direct-sequence codedivision multiple-access (MC DS-CDMA) using STS was proposed. This scheme shows good BER performance with small number of users and however, the performance of the system with larger MUI was not discussed. Recently, in [9], they adopted Hanzo's scheme [8], which shows a better result for larger number of users, but both transmitter and receiver designs are complicated.

In this article, we propose an open-loop MIMO OFDM-CDMA system using space, time, and frequency (STF) spreading [10]. The main goal is to achieve higher diversity gains and increased throughput by independently spreading data in STF with reasonable complexity. In addition, the system allows flexible data rates and efficient user multiplexing which are required for next generation wireless communications systems. An important advantage of using STF-domain spreading in MIMO OFDM-CDMA is that the maximum number of users supported is linearly proportional to the product of the S-domain, $\mathrm{T}$-domain and the F-domain spreading factors. Therefore, the MIMO OFDM-CDMA system using STF-domain spreading is capable of supporting a significantly higher number of users than other schemes using solely T-domain spreading. We will show through this article, that STF-domain spreading has significant throughput gains compared to conventional schemes. Furthermore, spreading on all the signal domains provides extra flexibility in user multiplexing and scheduling. In addition, it offers better diversity/multiplexing trade-off. The performance of MIMO OFDM-CDMA scheme using STF-domain spreading is investigated with zero-forcing (ZF) receiver. It is also shown that larger diversity gains can be achieved for a given number of users compared to other schemes. Moreover, higher number of users are able to share same channel resources, thus providing higher data rates than conventional techniques used in current HSPA+/LTE systems.

\section{System model}

In this section, joint space-time-frequency spreading is proposed for the downlink of an open-loop multi-user system employing single-user MIMO (SU-MIMO) system based on OFDM $\neg$ CDMA system.

\section{A. MIMO-OFDM channel model}

Consider a wireless OFDM link with $N_{f}$ subcarriers or tones. The number of transmit and receive antennas are $N_{t}$ and $N_{r}$, respectively. We assume that the channel has $L^{\prime}$ taps and the frequency-domain channel matrix of the $q$ th subcarrier is related to the channel impulse response as [11]

$$
\mathbf{H}_{q}=\sum_{l=0}^{L^{\prime}-1} \mathcal{H}(l) e^{\frac{-j 2 \pi l q}{N_{f}}}, \quad 0 \leq q<N_{f}-1,
$$

where the $N_{r} \times N_{t}$ complex-valued random matrix $\mathcal{H}(l)$ represents the $l$ th tap. The channel is assumed to be Rayleigh fading, i.e., the elements of the matrices $\mathcal{H}(l)\left(l=0,1, \ldots, L^{\prime}-1\right)$ are independent circularly symmetric complex Gaussian random variables with zero mean and variance $\sigma_{l}^{2}$, i.e., $[\mathcal{H}(l)]_{i j} \sim C N\left(0, \sigma_{l}^{2}\right)$. Furthermore, channel taps are assumed to be mutually independent, i.e., $E\left[\mathcal{H}(l) \mathcal{H}(k)^{*}\right]=0$, the path gains $\sigma_{l}^{2}$ are determined by the power delay profile of the channel.

Collecting the transmitted symbols into vectors $\mathbf{x}_{q}=\left[x_{q}^{(0)} x_{q}^{(1)} \ldots x_{q}^{\left(N_{t}-1\right)}\right]^{T}\left(q=0,1, \ldots, N_{f}-1\right) \quad$ with $x_{q}^{(i)}$ denoting the data symbol transmitted from the $i$ th antenna on the $q$ th subcarrier, the reconstructed data vector after FFT at the receiver for the $q$ th subcarrier is given by $[12,13]$

$$
\mathbf{y}_{q}=\sqrt{E_{s}} \mathbf{H}_{q} \mathbf{x}_{q}+\mathbf{n}_{q}, \quad k=0,1, \ldots, N_{f}-1,
$$

where

$\mathbf{y}_{q}=\left[y_{q}^{(0)} \gamma_{q}^{(1)} \ldots y_{q}^{\left(N_{r}-1\right)}\right]^{T}\left(q=0,1, \ldots, N_{f}-1\right) \quad$ with $\gamma_{q}^{(i)}$ denoting the data symbol received from the $j$ th antenna on the $q$ th subcarrier, $\mathbf{n}_{q}$ is complex-valued additive white Gaussian noise satisfying $E\left\{\mathbf{n}_{q} \mathbf{n}_{l}^{H}\right\}=\sigma_{n}^{2} \mathbf{I}_{N_{r}} \delta[q-l]$. The data symbols $x_{q}^{(i)}$ are taken from a finite complex alphabet and having unit average energy $\left(E_{s}=1\right)$.

\section{B. MIMO OFDM-CDMA system}

We will now focus on the downlink of a multi-access system that employs multiple antennas for MIMO OFDM-CDMA system. As shown in Figure 1a, the system consists of three different stages. The first stage employs the Joint Spatial, Time, and Frequency (STF) spreading which is illustrated in details in Figure 1b. The second stage is multi-user multiplexing (MUX) where all users are added together, and finally the third stage is IFFT to form the OFDM symbols. Then cyclic shifting is applied on each transmission stream. Specifically as shown in Figure 1, the IFFT outputs associated with the $i$ th transmit antenna are cyclicly shifted to the right by $(i-1) L$ where $L$ is a predefined value equal or greater to the channel length.

Now, we will describe in details the Joint STF spreading block shown in Figure 1b, where the signal is first spread in space, followed by time spreading and then 


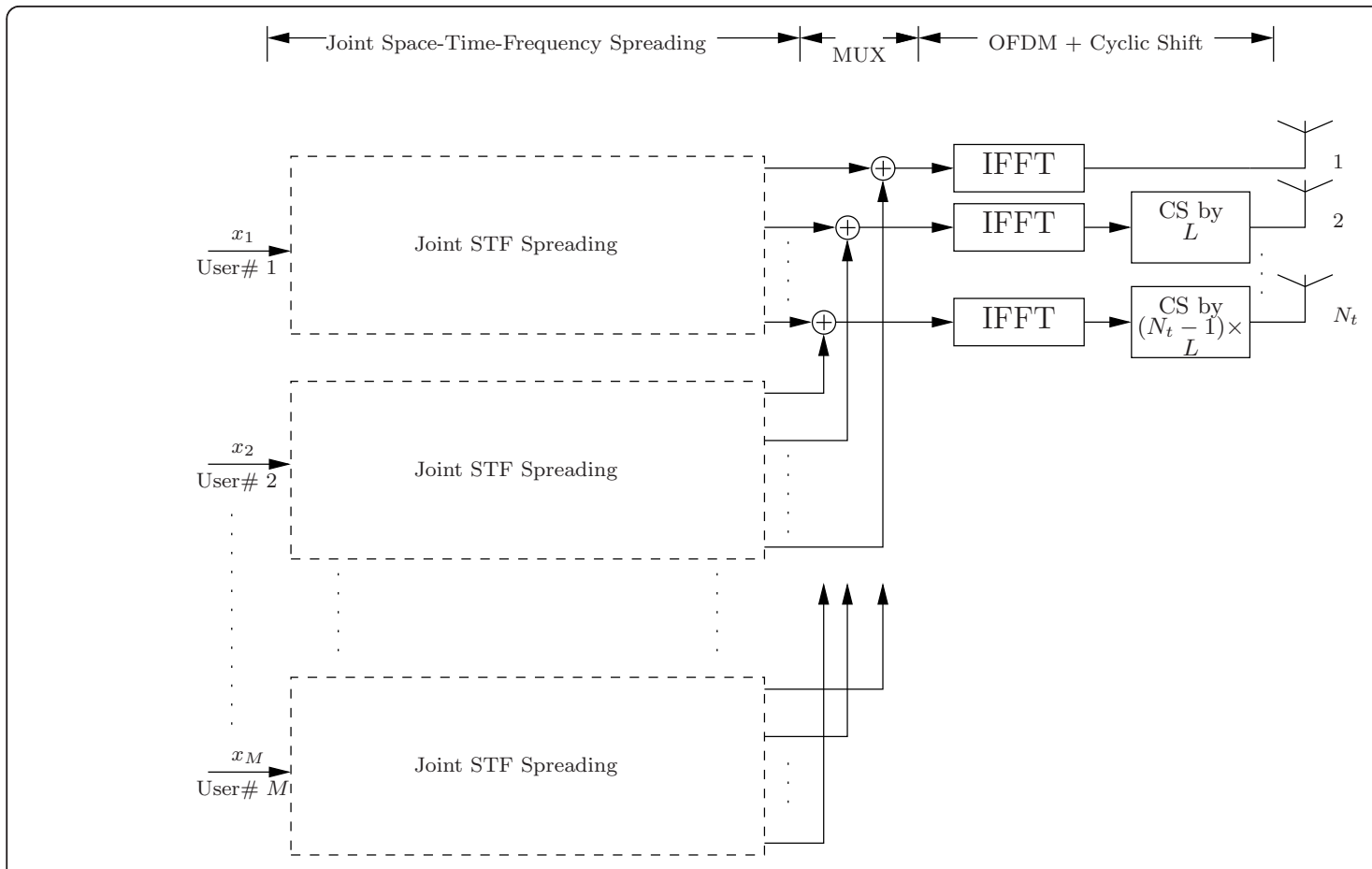

(a) MIMO OFDM-CDMA system

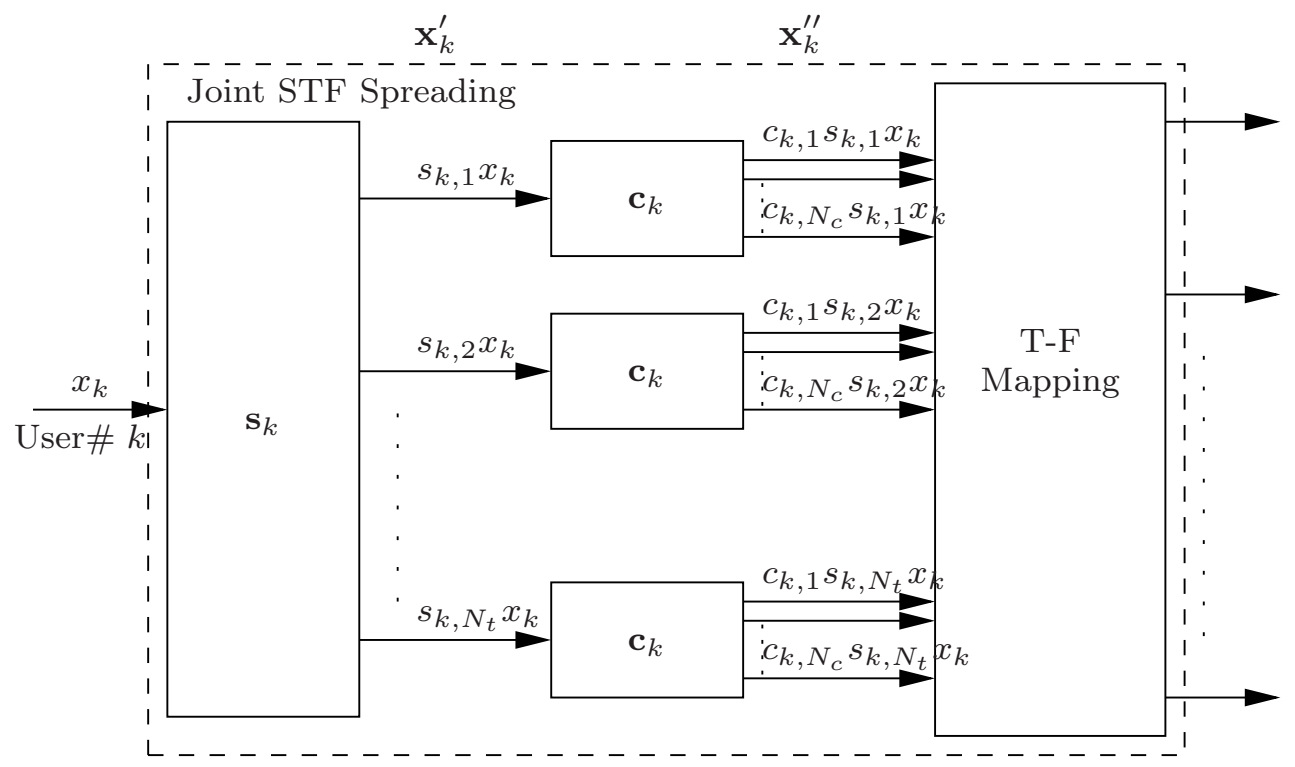

(b) Joint STF Spreading block diagram

Figure 1 MIMO OFDM-CDMA system block diagram.

time-frequency mapping is applied to ensure signal independency when transmitted and hence maximizing achievable diversity [14] on the receiver side.

\section{1) Spatial spreading}

Lets denote $x_{k}$ as the transmitted symbol from user $k$. It will be first spread in space domain using orthogonal code such as Walsh codes or columns of an FFT matrix of size $N_{t}$, as they are efficient short orthogonal codes. Let's denote $\mathbf{x}_{k}^{\prime}{ }_{k}$ as the spread signal in space for user $k$

$$
\begin{aligned}
\mathbf{x}_{k}^{\prime} & =\mathbf{s}_{k} x_{k} \\
& =\left[x_{k, 1}^{\prime}, x_{k, 2}^{\prime}, \ldots, x_{k, N_{t}}^{\prime}\right], \quad k=1,2, \ldots, M
\end{aligned}
$$


where $M$ is the number of users in the system, and $\mathbf{s}_{k}=\left[s_{k, 1}, s_{k, 2}, \ldots, s_{k, N_{t}}\right]^{T}$ is orthogonal code with size $N_{t}$ for user $k$.

\section{2) Time Spreading}

Then each signal in $\mathbf{x}_{k}^{\prime}$ is spread in time domain with $\mathbf{c}_{k}$ orthogonal code for user $k$ with size $N_{c}$. Let's denote $\mathbf{x}_{k}{ }_{k}$ as spread signal in time,

$$
\begin{aligned}
\mathbf{x}_{k, i}^{\prime \prime} & =\mathbf{c}_{k} x_{k, i}^{\prime} \\
& =\left[x^{\prime \prime}{ }_{k, i, 1}, x^{\prime \prime}{ }_{k, i, 2}, \ldots, x^{\prime \prime}{ }_{k, i, N_{c}}\right]^{T}, \quad i=1,2, \ldots, N_{t}
\end{aligned}
$$

where $x_{k, i, n}^{\prime \prime}$ is the transmitted signal for user $k$ from antenna $i$ at time $n$.

\section{3) Time-Frequency mapping}

The output of the space-time spreading is then mapped in time and frequency before IFFT. Figure 2 describes the Time-Frequency mapping method used in this system for user 1 at a particular transmit antenna. Without loss of generality all users will use the same mapping method at each antenna. Let's consider the mapping for $\mathbf{x}_{k, 1}$ and assume $x_{k, 1,1}^{\prime \prime}$ occupies OFDM symbol 1 at subcarrier $K_{1}, x_{k, 1,2}^{\prime \prime}$ occupies OFDM symbol 2 at subcarrier $K_{2}, \ldots$, and $x_{k, 1, N_{c}}^{\prime \prime}$ occupies OFDM symbol $N_{c}$ at subcarrier $K_{N_{c}}$. The next transmitted symbol $x_{k, 1,1}^{\prime \prime}$ occupies OFDM symbol 1 at subcarrier $K_{1}+1, x_{k, 1,2}^{\prime \prime}$ occupies OFDM symbol 2 at subcarrier $K_{2}+1, \ldots$, and $x_{k, 1, N_{c}}^{\prime \prime}$ occupies OFDM symbol $N_{c}$ at subcarrier $K_{N_{c}}+1$. Next symbols $x_{k, i}^{\prime \prime}$ are spread in the same manner as symbols 1 and 2 .

The assignment for each OFDM subcarrier is calculated from the fact that the IFFT matrix for our OFDM transmitted data for symbol 1 is $\mathbf{F}=\left[\mathbf{f}_{K_{1}}, \mathbf{f}_{K_{2}}, \ldots, \mathbf{f}_{K_{N_{c}}}\right]^{H}$ with size $N_{c} \times N_{f}$, where $\mathbf{F}^{H} \subset$ FFT matrix with size $N_{f}$. F matrix in this paper is a WIDE matrix $N_{c} \times N_{f}$ where the rows are picked from an FFT matrix and complex transposed (Hermitian). For this matrix to satisfy the orthogonality condition and to maintain independence, those rows needs to be picked as every $N_{f} / N_{c}$ column, so then and ONLY then, each column and row are orthogonal. The max rank cannot be more than $N_{c}$. The frequency spacing or jump introduced, made it possible to achieve the max rank, where each row and column is orthogonal within the rank. In order to achieve independent fading for each signal and hence maximizing frequency diversity, we need to have $\mathbf{F}^{H} \mathbf{F}=\mathbf{I} . \mathbf{F}^{H} \mathbf{F}=\mathbf{I}$ is only possible if $\mathbf{F}^{H}$ is constructed from every $N_{f} / N_{c}$ columns of the FFT matrix, $\mathbf{F}=\left[\mathbf{f}_{1}, \mathbf{f}_{N_{f} / N_{c}}, \mathbf{f}_{2 N_{f} / N_{c}}, \ldots, \mathbf{f}_{\left(N_{c}-1\right) N_{f} / N_{c}}\right]^{H}$. Therefore, if $K_{1}=1$, then $K_{2}=N_{f} / N_{c}, \ldots$, and $K_{N_{c}}=\left(N_{c}-1\right) N_{f} / N_{c}$.

\section{Receiver}

\section{A. Received signal of SU-MIMO system}

On the receiver side, let us consider the detection of symbol $x_{k}$ at receive antenna $j$. Let $\gamma_{K_{n}}^{(j)}$ be the received signal of the $K_{n}$-th subcarrier at the $j$-th receive antenna. Note that $K_{n}$ is the $K$-th subcarrier at time $n(n=1,2, \ldots$, $N_{c}$ ).

$$
\gamma_{K_{n}}^{(j)}=\mathbf{f}_{K_{n}}^{H}\left[\begin{array}{ccccc}
\mathbf{h}_{1, j} & \mathbf{0}_{L} & \ldots & \mathbf{0}_{L} \\
& \mathbf{0}_{L-L^{\prime}} & \vdots & \ldots & \vdots \\
\vdots & \mathbf{h}_{2, j} & \ddots & \mathbf{0} \\
\vdots & \mathbf{0}_{L-L^{\prime}} & \ddots & \mathbf{0} \\
& \vdots & \vdots & \vdots & \mathbf{0} \\
& \vdots & \ddots & \mathbf{h}_{N_{t}, j} \\
\mathbf{0} & \mathbf{0} & \ldots & \mathbf{0}_{N_{f}-\left(N_{t}-1\right) L-L^{\prime}}
\end{array}\right] .
$$

Stacking $y_{K_{n}}^{(j)}$ in one column, we have

$$
\underbrace{\left[\begin{array}{c}
y_{K_{1}}^{(j)} \\
\vdots \\
y_{K_{n}}^{(j)} \\
\vdots \\
y_{K_{N_{c}}}^{(j)}
\end{array}\right]}_{\mathbf{y}^{(j)}}=\underbrace{\left[\begin{array}{c}
\mathbf{f}_{K_{1}}^{H} c_{k, 1} \\
\vdots \\
\mathbf{f}_{K_{t}}^{H} c_{k, n} \\
\vdots \\
\mathbf{f}_{K_{N_{c}}}^{H} c_{k, N_{c}}
\end{array}\right]}_{\mathbf{F}_{c}} \underbrace{\left[\begin{array}{c}
\mathbf{h}_{1, j} s_{k, 1} \\
\mathbf{0}_{L-L^{\prime}} \\
\mathbf{h}_{2, j} s_{k, 2} \\
\mathbf{0}_{L-L^{\prime}} \\
\vdots \\
\mathbf{h}_{N_{t}, j} s_{k, N_{t}} \\
\mathbf{0}_{N_{f}-\left(N_{t}-1\right) L-L^{\prime}}
\end{array}\right]}_{\mathbf{h}_{j}^{s}}
$$

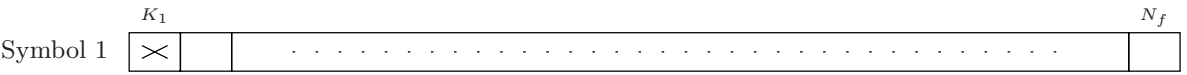

$$
\begin{aligned}
& \text { Symbol } 2 \text { L }
\end{aligned}
$$

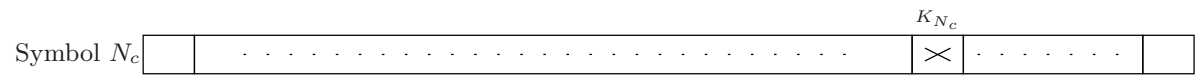


Here, $f_{K_{n}}$ stands for the $K_{n}$-th column of the $\left(N_{f} \times N_{f}\right)$ FFT matrix, $L$ is the cyclic shift on each antenna where $L>L^{\prime}$ ( $L^{\prime}$ is the channel length), and $\mathbf{h}_{i, j}$ is the impulse response from the $i$-th transmit antenna to the $j$-th receive antenna. Here, cyclic shifting in time has transformed the effective channel response $j$-th receive antenna to $\mathbf{h}_{j}^{s}$ as shown in Equation (6) instead of the addition of all channel responses. This will maximize the number of degrees of freedom from 1 to $N_{t}$.

In our scheme, we assumed that all users transmit on same time and frequency slots. As shown in Figure 1, we have the ability to achieve flexible scheduling in both time and frequency. This will contribute in more flexible system design for next-generation wireless systems as compared to other schemes.

\section{B. Achievable Diversity in SU-MIMO}

Let us assume that $x$, and $x^{\prime}$ are two distinct transmitted symbols from user $k$, and $\mathbf{y}^{(j)}, \mathbf{y}^{(j)}$ are the corresponding received signals at receive antenna $j$, respectively. To calculate diversity, we first calculate the expectation of the Euclidian distance between the two received signals $E\left[|| \mathbf{y}^{(j)}-\mathbf{y}^{(j)} \|^{2}\right]$, where $\mathbf{y}^{(j)}$ is defined by Equation (6),

$$
\begin{aligned}
E\left[\left\|\Delta \mathbf{y}^{(j)}\right\|^{2}\right] & =E\left[\| \mathbf{F}_{c} \mathbf{h}_{j}^{s}||^{2}|\Delta x|^{2}\right] \\
& =E\left[\mathbf{h}_{j}^{s H} \mathbf{F}_{c}^{H} \mathbf{F}_{c} \mathbf{h}_{j}^{s}|\Delta x|^{2}\right] \\
& =E\left[\mathbf{h}_{j}^{s H} \tilde{\mathbf{F}}_{c} \mathbf{h}_{j}^{s}|\Delta x|^{2}\right]
\end{aligned}
$$

In Equation (7), $\tilde{\mathbf{F}}_{c}$ is a toeplitz matrix $\left(N_{f} \times N_{f}\right)$ where it is all zero matrix except for the $r$ where $r=\sum_{t=1}^{N_{c}}\left|c_{k, n}\right|^{2}$, and all non-zero values are spaced $N_{c}$ entries apart, where

$$
\begin{aligned}
& \tilde{\mathbf{F}}_{c}=\left[\begin{array}{ccc}
1 & \ldots & 1 \\
\vdots & \ddots & \vdots \\
1 & \ldots & 1
\end{array}\right] \otimes\left[\begin{array}{lll}
r & & 0 \\
& \ddots & \\
0 & & r
\end{array}\right] \\
& =\mathbf{1}_{N_{f} / N_{c}} \otimes r \mathbf{I}_{N_{c}}
\end{aligned}
$$

The rank of the $\tilde{\mathbf{F}}_{c}$ matrix is found as,

$$
\operatorname{rank}\left(\tilde{\mathbf{F}}_{c}\right)=N_{c}
$$

Since the maximum achievable degrees of freedom for the transmitter is equal to $N_{t} L^{\prime}$, diversity can be found as $d=\min \left(N_{c}, N_{t} L^{\prime}\right)$ [15]. For this reason, in order to achieve maximum spatial diversity, we need to choose time spreading length $N_{c} \geq N_{t} L^{\prime}$.

\section{Receiver Design}

Now, let's assume all the users send data simultaneously where each user is assigned different spatial spreading code $\mathbf{s}_{k}$ and time spreading code $\mathbf{c}_{k}$ generated from a
Walsh-Hadamard function.

$$
\mathbf{y}_{K_{n}}=\sum_{k=1}^{M}\left(\mathbf{H}_{K_{n}} c_{k, n} \mathbf{s}_{k}\right) x_{k}+\mathbf{n}_{K_{n}}, \quad 1 \leq K_{n} \leq N_{f}
$$

where $k$ stands for user index and $K_{n}$ is the $K$-th subcarrier at time $n\left(n=1,2, \ldots, N_{c}\right)$.

Stacking $\mathbf{y}_{K_{n}}$ in one column, we have

$$
\begin{aligned}
\underbrace{\left[\begin{array}{c}
\mathbf{y}_{K_{1}} \\
\mathbf{y}_{K_{2}} \\
\vdots \\
\mathbf{y}_{K_{N_{c}}}
\end{array}\right]}_{\mathbf{y}}=\tilde{\mathbf{H}} \tilde{\mathbf{s}}_{1} x_{1}+\tilde{\mathbf{H}}_{\tilde{\mathbf{s}}_{2} x_{2}+\ldots+\tilde{\mathbf{H}} \tilde{\mathbf{s}}_{M} x_{M}+\mathbf{n}} \\
=\underbrace{\left[\begin{array}{llll}
\hat{\mathbf{H}}_{1} & \hat{\mathbf{H}}_{2} & \ldots & \hat{\mathbf{H}}_{M}
\end{array}\right]}_{\mathbf{G}} \mathbf{x}+\mathbf{n}
\end{aligned}
$$

where $\tilde{\mathbf{H}}$ is the modified channel matrix for the $N_{c}$ subcarriers, $\hat{\mathbf{H}}_{k}$ is the effective channel $\left(N_{c} N_{r} \times 1\right)$ for user $k$, and $\tilde{\mathbf{s}}_{k}=\mathbf{c}_{k} \otimes \mathbf{s}_{k}$ is the combined spatial-time spreading code, where

$$
\begin{aligned}
\tilde{\mathbf{H}} & =\operatorname{diag}\left\{\mathbf{H}_{K_{1}}, \mathbf{H}_{K_{2}}, \ldots, \mathbf{H}_{K_{N_{c}}}\right\} \\
\tilde{\mathbf{s}}_{k} & =\left[\begin{array}{c}
c_{k, 1} \mathbf{s}_{k} \\
c_{k, 2} \mathbf{s}_{k} \\
\vdots \\
c_{k, N_{c}} \mathbf{s}_{k}
\end{array}\right]
\end{aligned}
$$

At the receiver, the despreading and combining procedure with the time-frequency spreading grid pattern corresponding to the transmitter can not be processed until all the symbols within one super-frame are received. Then by using a MMSE or ZF receiver, data symbols could be recovered for all users $[16,17]$

$$
\begin{aligned}
& \hat{\mathbf{x}}=\left(\mathbf{G}^{H} \mathbf{G}+\sigma^{2} \mathbf{I}\right)^{-1} \mathbf{G}^{H} \mathbf{y} \quad \text { (MMSE) } \\
& \hat{\mathbf{x}}=\left(\mathbf{G}^{H} \mathbf{G}\right)^{-1} \mathbf{G}^{H} \mathbf{y} \quad(\mathrm{ZF})
\end{aligned}
$$

where $\hat{\mathbf{x}}=\left[\hat{x}_{1}, \hat{x}_{2}, \ldots, \hat{x}_{M}\right]$, and $M$ is the number of users.

\section{Performance Evaluation for Zero Forcing Receiver}

In this section, we will calculate probability of bit error for Zero-Forcing receiver (ZF) $[18,19]$ to examine the performance of our space-time-frequency spreading. ZF is considered in our paper, because of its simpler design. $\mathrm{ZF}$ is more affordable in terms of computational complexity and lower cost. As well, the impact of noise enhancement from ZF is reduced due to the inherent 
property of avoiding poor channel quality using space, time and frequency spreading. Without the loss of generality, the signal from first user is regarded as the desired user and the signals from all other users as interfering signals. With coherent demodulation, the decision statistics of user 1 symbol is given as,

$$
\begin{aligned}
\hat{x}_{1} & =\left(\hat{\mathbf{H}}_{1}^{H} \hat{\mathbf{H}}_{1}\right)^{-1} \hat{\mathbf{H}}_{1}^{H} \mathbf{y} \\
& =\left(\tilde{s}_{1}^{H} \tilde{\mathbf{H}}^{H} \tilde{\mathbf{H}} \tilde{\mathbf{s}}_{1}\right)^{-1} \tilde{\mathbf{s}}_{1}^{H} \tilde{\mathbf{H}}^{H}\left(\tilde{\mathbf{H}} \tilde{\mathbf{s}}_{1} x_{1}+\tilde{\mathbf{H}} \tilde{\mathbf{s}}_{2} x_{2}+\ldots+\tilde{\mathbf{H}} \tilde{\mathbf{s}}_{M} x_{M}+\mathbf{n}\right)
\end{aligned}
$$

Then, the desired signal, multiple access interference (MAI) and the noise are $S, I, \eta$, respectively.

$$
\begin{aligned}
S & =x_{1} \\
I & =\left(\tilde{\mathbf{s}}_{1}^{H} \tilde{\mathbf{H}}^{H} \tilde{\mathbf{H}} \tilde{\mathbf{s}}_{1}\right)^{-1} \sum_{k=2}^{M}\left(\tilde{\mathbf{s}}_{1}^{H} \tilde{\mathbf{H}}^{H} \tilde{\mathbf{H}} \tilde{\mathbf{s}}_{k}\right) x_{k} \\
\eta & =\left(\tilde{\mathbf{s}}_{1}^{H} \tilde{\mathbf{H}}^{H} \tilde{\mathbf{H}} \tilde{\mathbf{s}}_{1}\right)^{-1} \tilde{\mathbf{s}}_{1}^{H} \tilde{\mathbf{H}}^{H} \mathbf{n}
\end{aligned}
$$

To compute signal-to-interference noise ratio (SINR), which is defined as $\Gamma$, we will assume $S, I, \eta$ are uncorrelated,

$$
\begin{aligned}
\Gamma_{\tilde{\mathrm{H}}} & =\frac{E\left|S^{2}\right|}{E\left[|\eta|^{2}\right]+E\left[|I|^{2}\right]} \\
& =\frac{E\left[S^{2}\right]}{\sigma_{I}^{2}+\sigma_{\eta}^{2}}
\end{aligned}
$$

where, $x_{k}$ (MAI) are assumed to be mutually independent, therefore input symbols $\left\{x_{k}\right\}_{k=1}^{M}$ are assumed Gaussian with unit variance. The expectation is taken over the user symbols $x_{k}, k=1, \ldots, M$ and noise $\mathbf{k}$.

Since the effective channel is denoted as $\hat{\mathbf{H}}_{n}=\tilde{\mathbf{H}} \tilde{\mathbf{s}}_{k}$, then

$$
\hat{\mathbf{H}}_{k}^{H} \hat{\mathbf{H}}_{l}=\tilde{\mathbf{s}}_{k}^{H} \tilde{\mathbf{H}}^{H} \tilde{\mathbf{H}} \tilde{\mathbf{s}}_{l}
$$

Desired signal average power is defined as,

$$
E\left[S^{2}\right]=1
$$

Multiple access interference (MAI) is defined as,

$$
\begin{aligned}
\sigma_{I}^{2} & =E\left[\left(\tilde{\mathbf{s}}_{1}^{H} \tilde{\mathbf{H}}^{H} \tilde{\mathbf{H}} \tilde{\mathbf{s}}_{1}\right)^{-2} \sum_{k=2}^{M}\left|\tilde{\mathbf{s}}_{1}^{H} \tilde{\mathbf{H}}^{H} \tilde{\mathbf{H}} \tilde{\mathbf{s}}_{k}\right|^{2}\right] \\
& =E\left[\left|\hat{\mathbf{H}}_{1}^{H} \hat{\mathbf{H}}_{1}\right|^{-2} \sum_{k=2}^{M}\left|\hat{\mathbf{H}}_{1}^{H} \hat{\mathbf{H}}_{k}\right|^{2}\right]
\end{aligned}
$$

where $\hat{\mathbf{H}}_{1}^{H} \hat{\mathbf{H}}_{k}$ is the projection of $\hat{\mathbf{H}}_{1}$ on $\hat{\mathbf{H}}_{k}$. Without loss of generality, let's assume in Equation (23) that
$\hat{\mathbf{H}}_{1}=\sqrt{\hat{\mathbf{H}}_{1}^{H} \hat{\mathbf{H}}_{k}} \mathbf{P e} e_{1}$, where $\mathbf{P}$ is any permutation matrix, and $\mathbf{e}_{1}$ is the 1-st column of the $\mathbf{I}$ identity matrix,

$$
\begin{aligned}
\sigma_{I}^{2} & =E\left[\left|\hat{\mathbf{H}}_{1}^{H} \hat{\mathbf{H}}_{1}\right|^{-2} \sum_{k=2}^{M}\left|\sqrt{\hat{\mathbf{H}}_{1}^{H} \hat{\mathbf{H}}_{1}} \mathbf{e}_{1}^{H}\left(\mathbf{P}^{H} \hat{\mathbf{H}}_{k}\right)\right|^{2}\right] \\
& =E\left[\left|\hat{\mathbf{H}}_{1}^{H} \hat{\mathbf{H}}_{1}\right|^{-1} \sum_{k=2}^{M}\left|\mathbf{e}_{1}^{H} \mathbf{P}^{H} \hat{\mathbf{H}}_{k}\right|^{2}\right] \\
& =\left(\frac{1}{M-1}\right) \sum_{k=2}^{M}\left|\hat{z}_{k}\right|^{2} /\left(\frac{1}{N_{t} N_{c}}\right) \sum_{m=1}^{N_{c} N_{t}}\left|\hat{x}_{m}\right|^{2}
\end{aligned}
$$

where $\left|\hat{z}_{k}\right|^{2}$ and $\left|\hat{x}_{m}\right|^{2}$ are chi-squared random variables, as Equation (21) shows that $\hat{\mathbf{H}}_{k}$ is gaussian random variable $\sim C N(0,1)$

Noise average power is defined as,

$$
\begin{aligned}
\sigma_{\eta}^{2} & =E\left\{\left(\tilde{\mathbf{s}}_{1}^{H} \tilde{\mathbf{H}}^{H} \tilde{\mathbf{H}} \tilde{\mathbf{s}}_{1}\right)^{-2} \tilde{\mathbf{s}}_{1}^{H} \tilde{\mathbf{H}}^{H} \mathbf{n n}^{H} \tilde{\mathbf{H}} \tilde{\mathbf{s}}_{1}\right\} \\
& =E\left[\left|\hat{\mathbf{H}}_{1}^{H} \hat{\mathbf{H}}_{1}\right|^{-2} \tilde{\mathbf{s}}_{1}^{H} \tilde{\mathbf{H}}^{H} \tilde{\mathbf{H}} \tilde{\mathbf{s}}_{1}\right] \sigma^{2} \\
& =E\left[\left|\hat{\mathbf{H}}_{1}^{H} \hat{\mathbf{H}}_{1}\right|^{-1}\right] \sigma^{2} \\
& =\sigma^{2} /\left(\frac{1}{N_{t} N_{c}}\right) \sum_{m=1}^{N_{c} N_{t}}\left|\hat{x}_{m}\right|^{2}
\end{aligned}
$$
by

Therefore, the probability of error can be simply given

$$
P(e)=Q(\sqrt{\Gamma})
$$

From Equations (22), (24), and (25), we can obtain SINR

$$
\begin{aligned}
& \Gamma=\frac{E\left[S^{2}\right]}{\sigma_{I}^{2}+\sigma_{\eta}^{2}} \\
& =\frac{1}{\frac{\left(\frac{1}{M-1}\right) \sum_{k=2}^{M}\left|\hat{z}_{k}\right|^{2}}{\left(\frac{1}{N_{t} N_{c}}\right) \sum_{m=1}^{N_{c} N_{t}}\left|\hat{x}_{m}\right|^{2}}+\frac{\sigma^{2}}{\left(\frac{1}{N_{t} N_{c}}\right) \sum_{m=1}^{N_{c} N_{t}}\left|\hat{x}_{m}\right|^{2}}} \\
& =\frac{1}{\left(1 / F_{a, b}\right)+\left(\sigma^{2} / \chi^{2}\right)}
\end{aligned}
$$

where $F_{a, b}$ is $\mathrm{F}$-distribution random variable (ratio between two chi-squared random variables) where $a=$ $N_{t} N_{c}$ and $b=M-1$ degrees of freedom, and $\chi^{2}$ is chisquared random variable with $N_{t} N_{c}$ degrees of freedom. It is clear that when interference is small enough, the most dominant part will be the $\chi^{2}$ which agrees with Raleigh fading channel where no MUI exists. When the 
MUI dominates channel noise, Equation (27) can be approximated as $\Gamma=F_{a, b}$

Now, by assuming all users are scheduled to transmit at similar symbol rates $R_{s}$ at a time instance, we could calculate BER using Equation (26) by statistically averaging over the probability density function of $F_{a, b}$ (see Appendix), i.e., by substituting Equation (27) in Equation (26).

$$
\begin{aligned}
P_{e} & =\int p\left(F_{a, b}\right) Q\left(\sqrt{\Gamma_{F_{a, b}}}\right) d F_{a, b} \\
& \leq \frac{\left(P / \sigma^{2}\right)^{b} a^{a} b^{b}}{\beta(b, a)} \int_{0}^{\infty} \frac{\gamma^{a-1}}{\left(\left(P / \sigma^{2}\right) b+a y\right)^{a+b}}\left(\frac{1}{6} e^{-\gamma}+\frac{1}{2} e^{-\frac{4}{3} \gamma}\right) d y
\end{aligned}
$$

In Equation (28) $y$ is SINR defined in Equation (27), $P / \sigma^{2}$ is the signal-to-noise ratio (SNR), $a$ is equal to $N_{t} N_{c}$, and $b=M-1$.

In Figure 3, we compare the SINR PDFs for our proposed scheme defined by Equation (27) and 2D OFDMCDMA [6]. It is clear that the probability of SINR has higher values in our proposed OFDM-CDMA system compared to 2D OFDM-CDMA system, which means that the average SINR for our proposed system will be more likely to be higher than that of the 2D OFDMCDMA system. This is confirmed by numerically evaluating $P($ SINR $<20 \mathrm{~dB})$ for our proposed system and $2 \mathrm{D}$ OFDM-CDMA system, which are 0.6479 and 0.5468 respectively. This improvement will lead to better multiuser diversity gains. In Figure 4, the PDF curves of the proposed scheme with various number of users are provided. From Figures 3 and 4, it can be seen that the SINR PDF curve of the proposed scheme with 32 users is close to that of the $2 \mathrm{D}$ scheme with 16 users. This shows that the proposed scheme supports twice the number of users in a system with 4 transmit and 4 receive antennas. It is also interesting to note that the simulated results match well with our analytical results provided by Equation (27). Figure 4 shows that the average SINR is $20 \mathrm{~dB}$ for all users, and the most probable SINR decreases as the number of users increases.

\section{E. Complexity}

The process of spreading each bit on space, time and frequency in a parallel manner was considered to be a complicated issue [20]. However, the proposed OFDMCDMA has efficient mapping in bit allocation in space, time and frequency without degrading overall system performance, and therefore it is less complex. In other OFDM-CDMA systems, RAKE receiver is widely used to take advantage of the entire frequency spread of a particular bit, that adds to overall system hardware complexity. In our proposed open-loop MIMO OFDMCDMA, RAKE receiver is not needed as each bit is spread in time and frequency, occupying different time and frequency slots, where each bit is spread to ensure frequency independence as shown in Figure 2. Also, other systems that use space-time-frequency (STF) coding as in [16], has more complexity than our proposed system. Their spreading technique uses space-time block

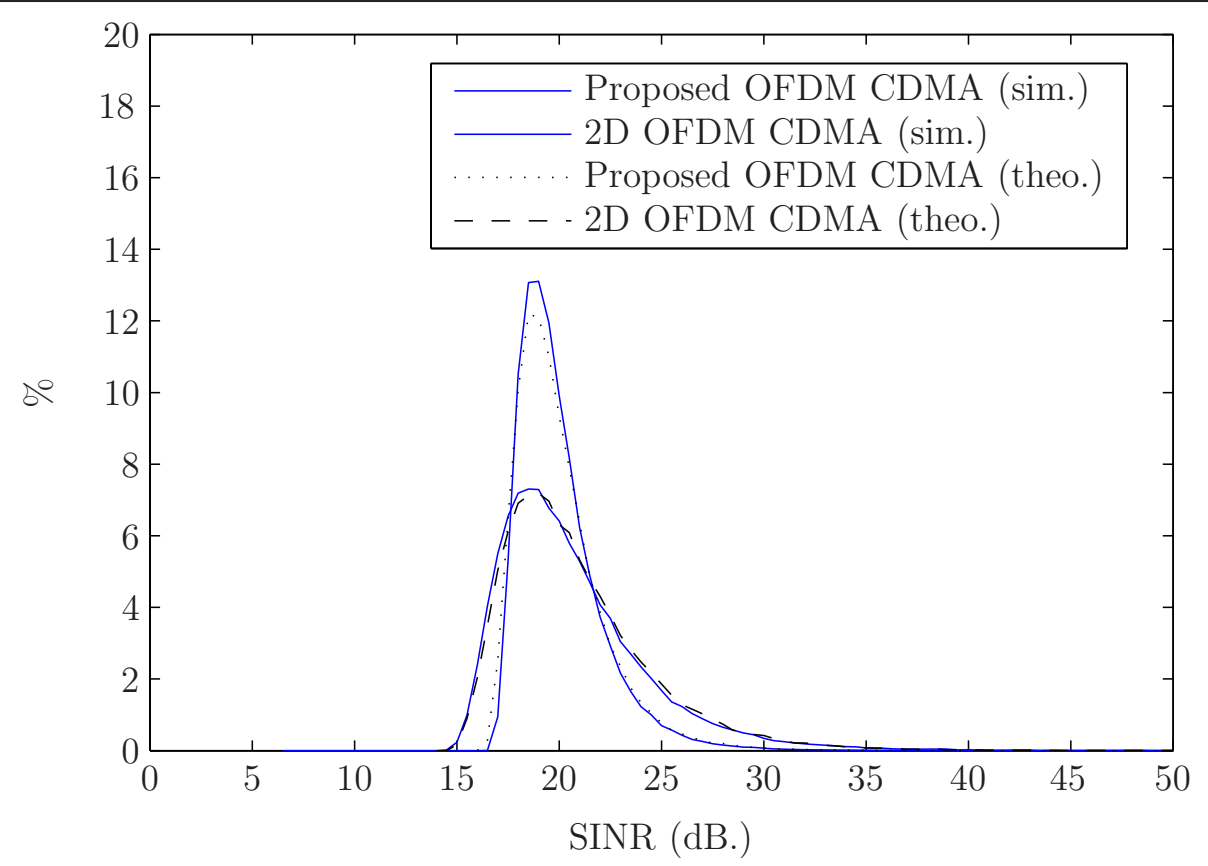

Figure 3 Probability density function for SINR for $E_{s} / \sigma^{2}=20 \mathrm{~dB}$ for our proposed scheme (solid) and 2D OFDM-CDMA (dotted), for both simulated and calculated $\left(N_{t}, N_{r}=4, N_{c}=16\right.$, and $\left.M=16\right)$. 


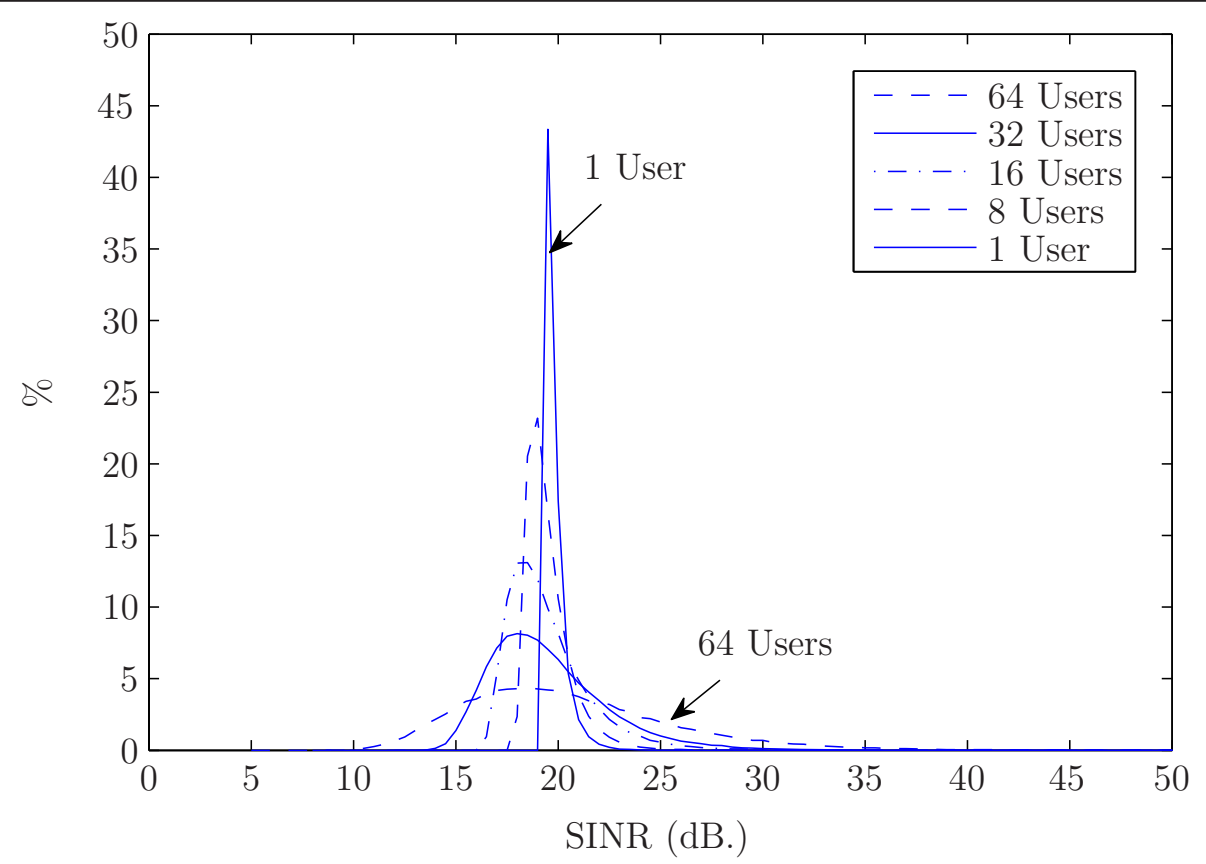

Figure 4 Probability density function for SINR for $E_{s} / \sigma^{2}=20 \mathrm{~dB}$ for our proposed scheme with different number of users.

codes or space-time trellis codes and then uses subcarrier selectors to map signals to different OFDM frequency subcarriers. Our proposed STF spreading method does not involve coding or precoding, just bit spreading to maintain signal orthogonality and maximize diversity at receiver side. Figure 5 shows that our proposed system has better performance than [16], by improving both diversity and coding gains.

\section{Simulation results}

Computer simulations were carried out to investigate the performance gain of the proposed open-loop MIMO OFDM-CDMA system with joint space-frequency-time spreading. The channel is a multipath channel modelled as a finite tapped delay line with $L=4$ Rayleigh fading paths. Walsh-Hadamard (WH) codes are utilized for both space and time spreading. Different codes are

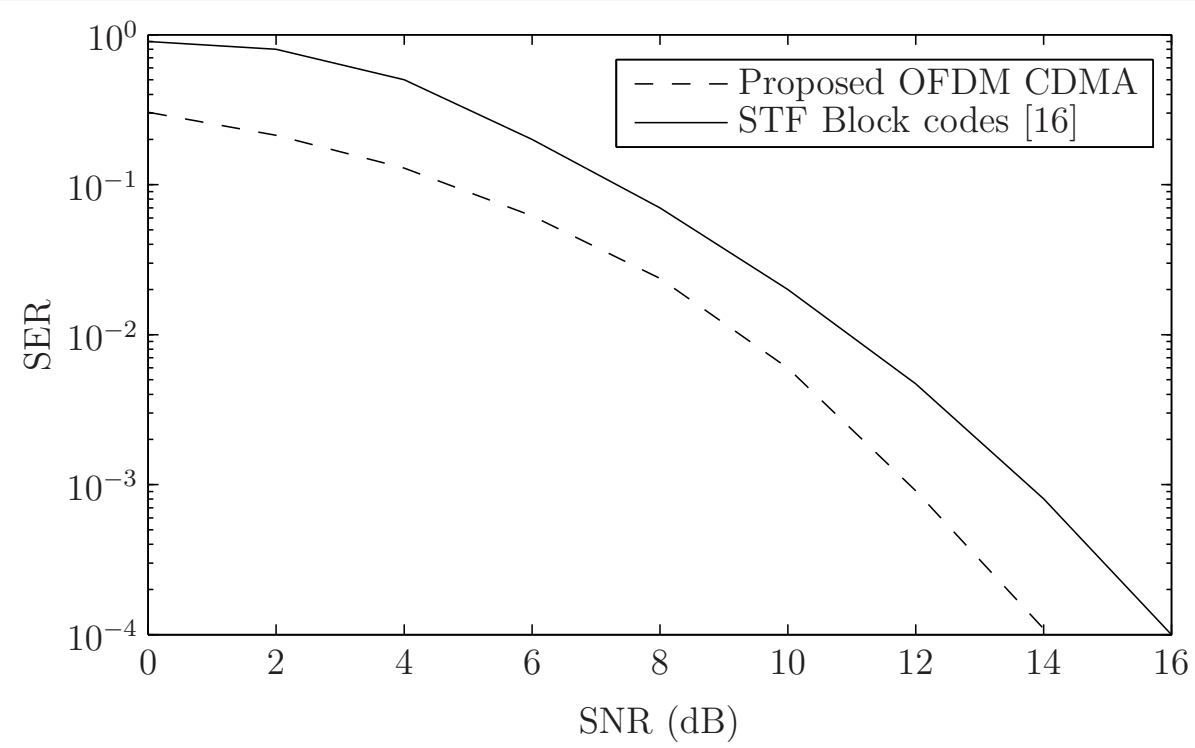

Figure 5 SER vs SNR comparison of the proposed OFDM-CDMA scheme (dotted) and 2D STF block codes [16](solid) with 2Tx, 1Rx, $N_{f}$ $=64, L=4$ (multiray channels). 
assigned to different users. The OFDM super-frame contains 16 OFDM symbols, which is equal to the length of the time spreading code $N_{c}=16$, where each OFDM symbol has 128 subcarriers. The channel estimation is assumed to be perfect, quadrature phase-shift keying (QPSK) constellation is used. We assume a MIMO channel with $N_{t}=4$ transmit antennas and $N_{r}=$ $1,2,4$ receive antennas. It is assumed that the mean power of each interfering user is equal to the mean power of the desired signal. The maximum number of users allowed by the system is $N_{c}\left(\min \left(N_{t}, N_{r}\right)\right)$.

Figure 6 shows the Bit error rate (BER) performance of OFDM-CDMA versus the average $E_{s} / N_{0}$ with different number of active users with slow fading channel for 4 transmit and 4 receive antennas, where the solid lines stand for our proposed scheme, while the dotted line stands for the double-orthogonal coded (DOC)-STFSCDMA scheme proposed in [9]. It is clear that our scheme has better resiliency to the frequency selectivity of the channel due to the inherent property of avoiding poor channel quality using the proposed space, time and frequency spreading.

Figure 7 shows the Block error rate (BLER) performance of OFDM-CDMA versus the average $E_{s} / N_{0}$ with different number of active users with slow fading channel for 4 transmit and 4 receive antennas, where the solid lines stand for our proposed scheme, while the dotted line stands for the 2D OFDM-CDMA. It is obvious that when we spread our signal on space, time, and frequency, we had better performance as we were able to maintain maximum achievable spatial diversity on the receiver side.

Figures 8 and 9 show the BER performance of OFDMCDMA versus the average $E_{b} / N_{0}$ for 1 and 2 receive antennas, respectively. In our simulations, we compare our proposed scheme with 2D OFDM-CDMA described in [6]. The maximum number of users allowed in Figures 8 and 9 are 16, and 32 users, respectively. Simulation results show that our proposed system has better performance, but as the number of users increases to max, diversity advantages are decreased due to the fact of diversity/multiplexing trade-off. On the other hand, when we decrease the number of receive antennas to one, our proposed scheme is superior because we are able to maintain maximum possible spatial diversity on the receiver side, but the other scheme is not able to compensate when reducing the number of receive antennas to one. Comparing both figures, our scheme has greater gains when reducing receive antennas from 2 to 1 , offering better diversity/multiplexing trade-off. Also, Figure 8 confirms that the results shown for SINR pdf in Figure 3 holds for 1 receive antenna, as BER curves for the 2D OFDM-CDMA with 4 users coincides with our proposed system but with 8 users. Therefore, our proposed scheme has twice the throughput with the same BER performance.

Figure 10 shows system user throughput. The proposed system is able to have higher number of users

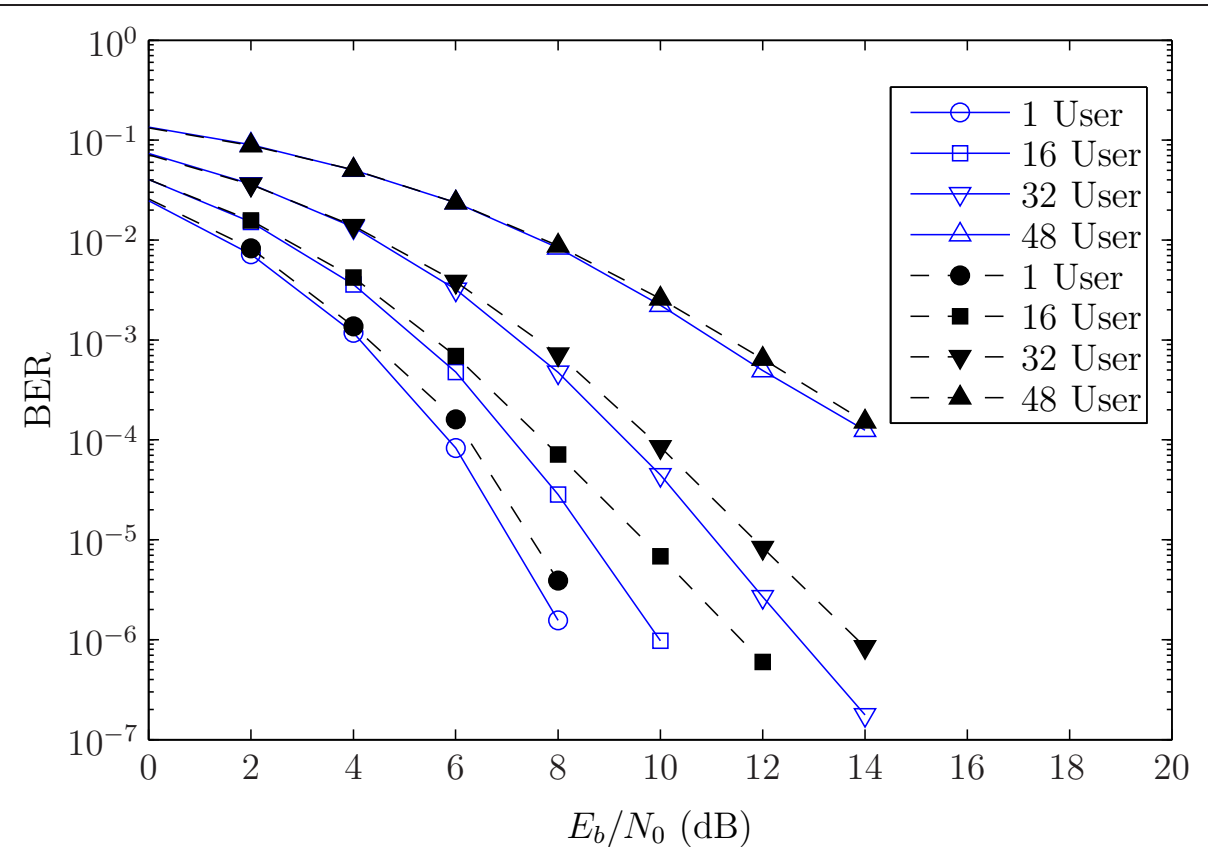

Figure 6 BER comparison for OFDM-CDMA system with 4Tx, 4Rx with our proposed scheme (solid) and DOC-STFS-CDMA [9](dotted) in a slow fading frequency-selective environment. 


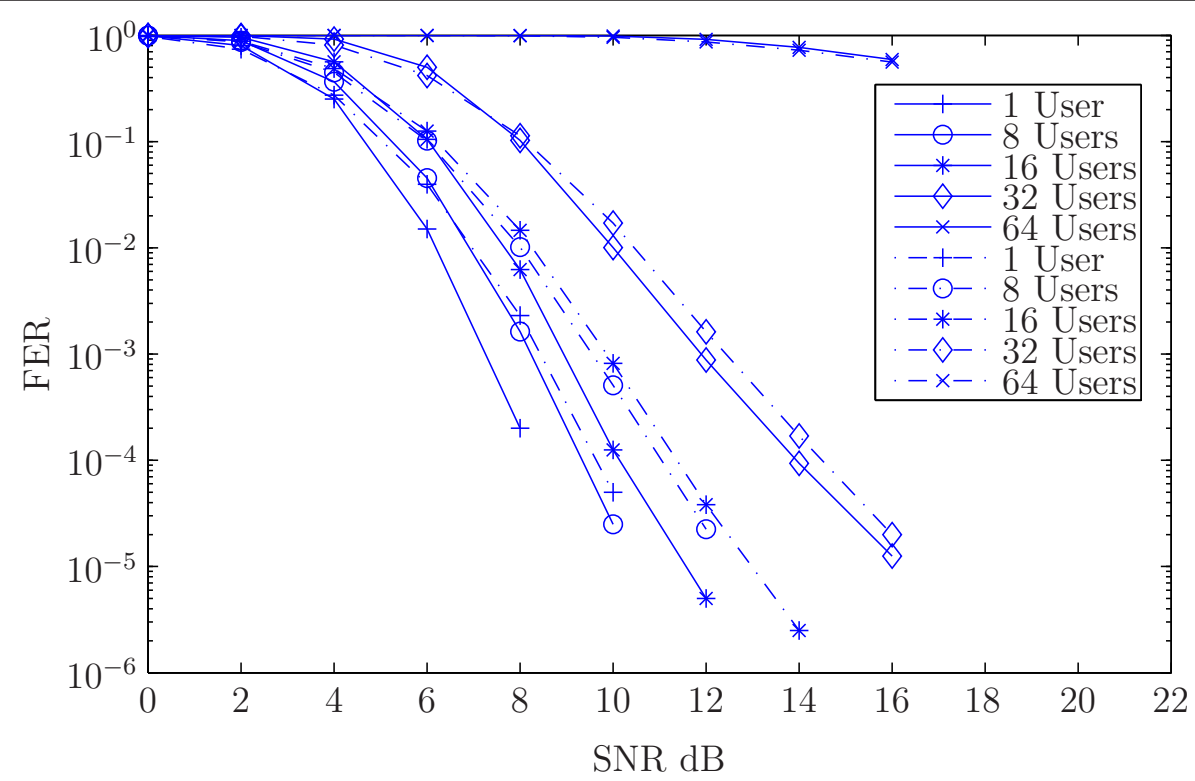

Figure 7 BLER comparison for OFDM-CDMA system with 4Tx, 4Rx with our proposed scheme (solid) and 2D OFDM-CDMA (dotted) in a slow fading frequency-selective environment.

because we are able to fully exploit the spatial dimension of the channel. This leads to lower BLER, and higher diversity gains, that will contribute to increased number of users without degrading the system performance as shown in the SINR pdf graphs in Figure 3. The system is able to maintain reliable communication with reasonable super-frame drops up to 32 users, as compared to 2D OFDM-CDMA. Also, we are able to maintain double number of users with same BLER performance. At 32 users, the system is able to fully utilize the channel at $\mathrm{SNR}=10 \mathrm{~dB}$.

In Figure 11, we compare the upper-bound result in Equation (28) with simulation result. It is clear that the tight bound we proposed matches our simulated results perfectly.

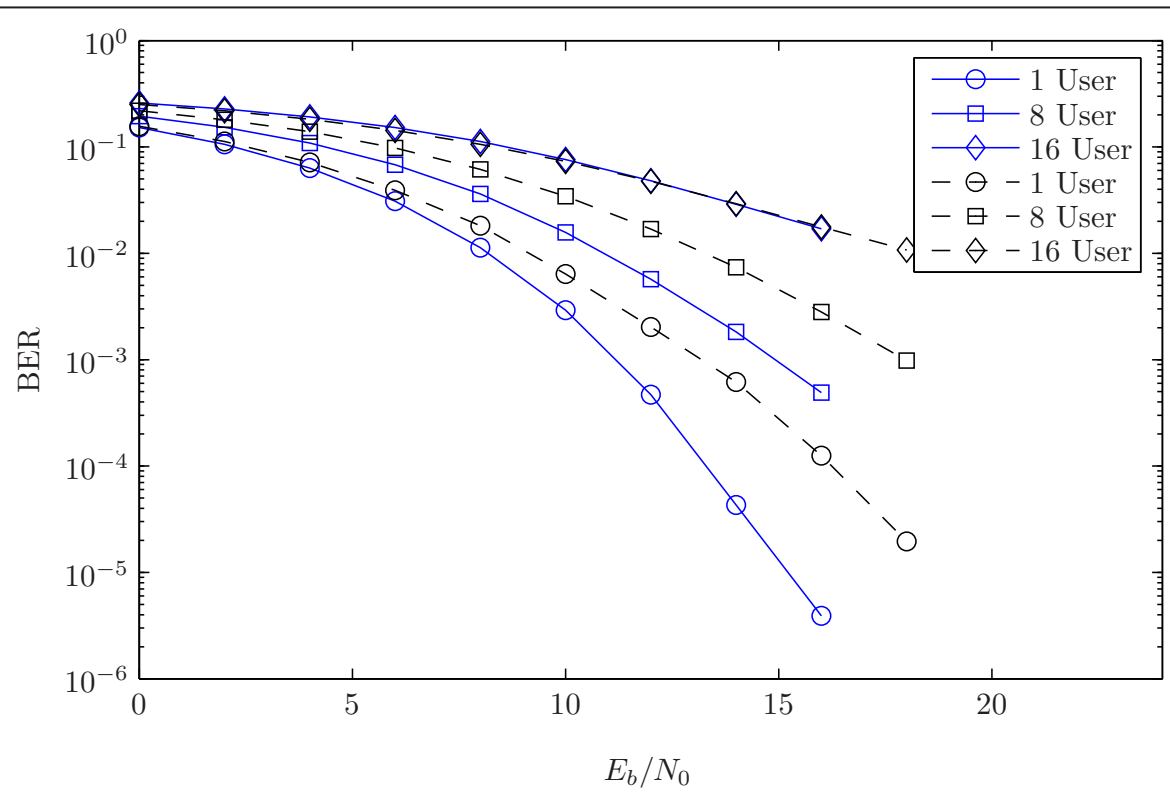

Figure 8 BER comparison for OFDM-CDMA system with 4Tx, 1Rx of the proposed scheme (solid) and 2D OFDM-CDMA (dotted) in a slow fading frequency-selective environment. 


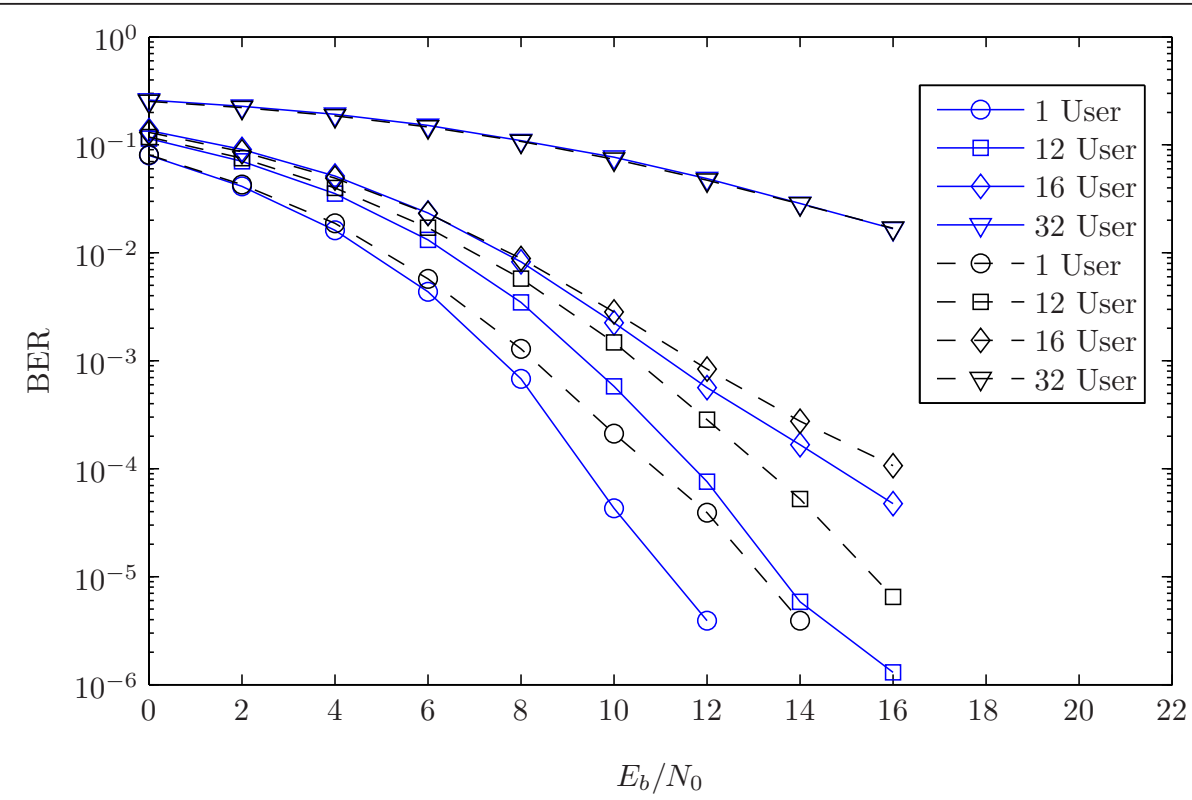

Figure 9 BER comparison for OFDM-CDMA system with 4Tx, 2Rx of the proposed scheme (solid) and 2D OFDM-CDMA (dotted) in a slow fading frequency-selective environment.

\section{Conclusion}

In this paper, we have proposed an open-loop MIMO OFDM-CDMA scheme using space-time-frequency spreading (STFS), in the presence of frequency-selective Rayleigh-fading channel. The BER and BLER performance of the OFDM-CDMA system using STFS has been evaluated taking into consideration diversity/ multiplexing trade-off over frequency-selective Rayleighfading channels.

We showed that our proposed system gives the advantage of maintaining maximum achievable spatial diversity on the receiver side in the case of slow frequency-selective Rayleigh-fading channels. Also, by appropriately selecting the system parameters $N_{t}$, and $N_{c}$, the OFDM-CDMA

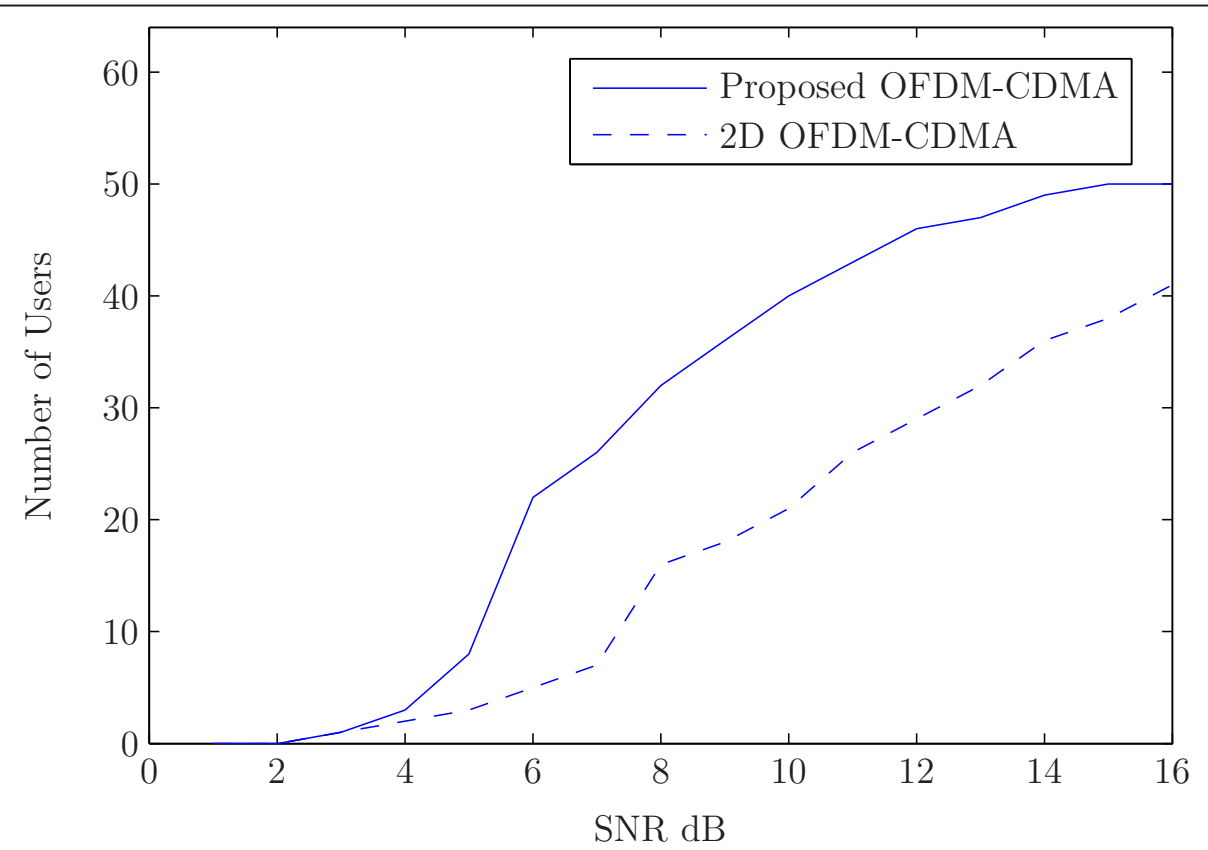

Figure 10 System throughput comparison for OFDM-CDMA system with 4Tx, 4Rx of the proposed scheme (solid) and 2D OFDMCDMA (dotted) in a slow fading frequency-selective environment. 


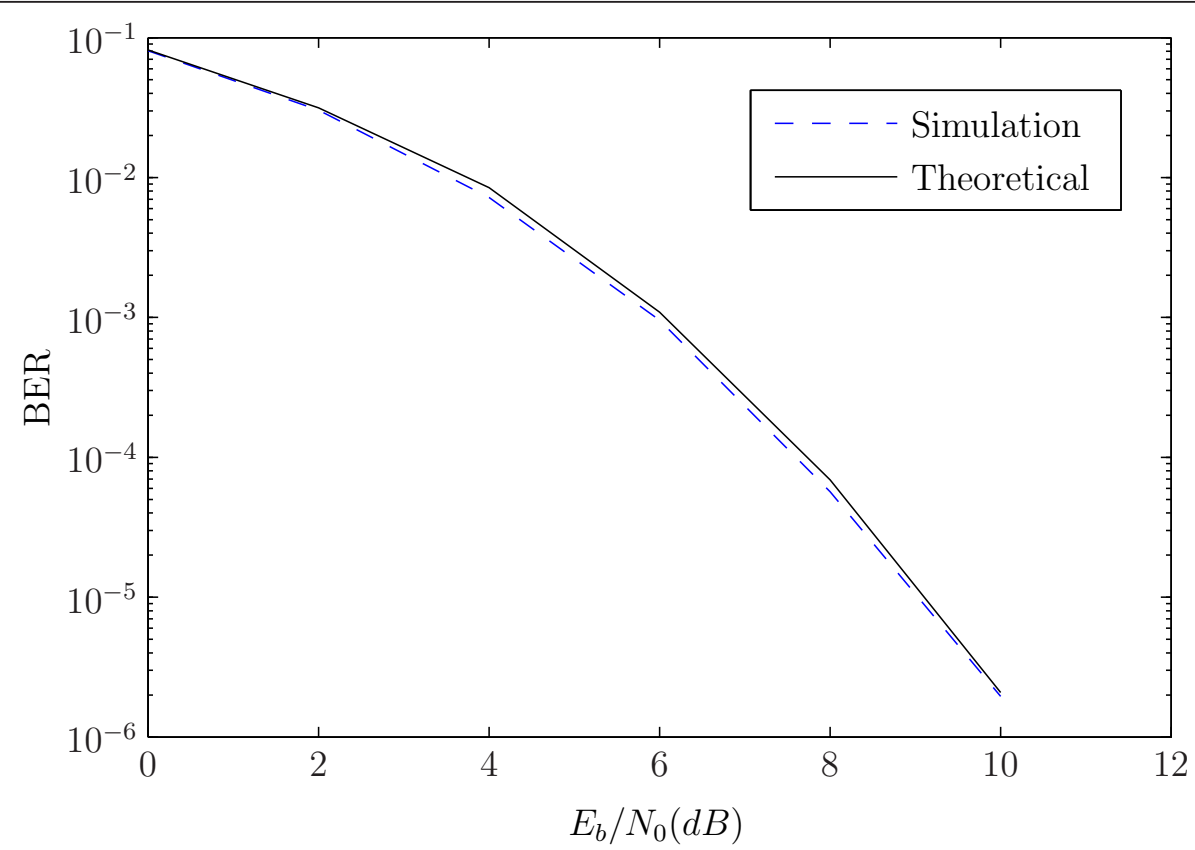

Figure 11 Probability of error for analytical (solid) vs simulation (dotted).

system using STFS is rendered capable of achieving higher number of users than other schemes. System throughput has increased as our proposed system was capable of achieving higher SINR than other schemes at similar SNRs. Higher diversity gains than other systems were shown, when number of receive antennas are reduced to one, as our system was able to maximize the number of degrees of freedom, by exploiting the spatial dimension of the channel. Our system showed great improvements, in system performance and throughput compared to other systems without sacrificing complexity.

\section{Appendix}

\section{Upper bound for $P_{e}$}

In this section, we will show the numerical evaluation that led to Equation (28). When $M$ is large enough, the interference component will be the dominant component, $\left(\frac{1}{M-1}\right) \sum_{k=2}^{M}\left|\hat{z}_{k}\right|^{2}>\sigma^{2}$ and Equation (27) can be expressed as follows

$$
\operatorname{SINR}=y=\frac{P / \sigma^{2}}{(1 / x)}
$$

where $x$ is $f_{a, b}$-distribution with $a=N_{t} N_{c}$ and $b=M$ 1 degrees of freedom, the probability density function $f_{a}$, $b(x)$ is defined as

$$
f_{a, b}(x)=\frac{a^{a} b^{b}}{\beta(a, b)} \frac{x^{a-1}}{(b+a x)^{a+b}}
$$

Substituting Equation (30) into Equation (29), we obtain the probability density function for SINR as,

$$
f(y)=\left(\frac{\left(P / \sigma^{2}\right)^{b} a^{a} b^{b}}{\beta(b, a)}\right) \frac{\gamma^{a-1}}{\left(\left(P / \sigma^{2}\right) b+a \gamma\right)^{a+b}}
$$

As mentioned earlier, probability of error is defined as,

$$
P_{e}=\int_{0}^{\infty} f(y) Q(\sqrt{y}) d y
$$

In [21], it was shown that $\operatorname{erfc}($.$) can be approximated$ to a tighter bound than Chernoff-Rubin bound,

$$
Q(\sqrt{y}) \leq \frac{1}{6} e^{-\gamma}+\frac{1}{2} e^{-\frac{4}{3} \gamma}
$$

By substituting Equations (31) and (33) into Equation (32), we obtain the probability of error $P_{e}$,

$$
P_{e} \leq \frac{\left(P / \sigma^{2}\right)^{b} a^{a} b^{b}}{\beta(b, a)} \int_{0}^{\infty} \frac{\gamma^{a-1}}{\left(\left(P / \sigma^{2}\right) b+a y\right)^{a+b}}\left(\frac{1}{6} e^{-\gamma}+\frac{1}{2} e^{-\frac{4}{3} \gamma}\right) d y
$$

\section{Competing interests}

The authors declare that they have no competing interests.

Received: 12 February 2011 Accepted: 23 December 2011 Published: 23 December 2011 


\section{References}

1. 3rd Generation Partnership Project; Technical Specification Group Radio Access Network; Physical layer-General description, 3GPP TS 25.201, (May 2008)

2. R Prasad, S Hara, An overview of multi-carrier CDMA. in Proc IEEE 4th Int Symp Spread Spectrum Techniques and Applications, Mainz, 107-114 (September 1996)

3. S Kaiser, K Fazel, A flexible spread-spectrum multi-carrier multiple-access system for multi-media applications. in Proc 1997 Int Symp Personal, Indoor and Mobile Radio Communications, Helsinki Finland, 100-104 (September 1997)

4. S Kaiser, OFDM code-division multiplexing in fading channels. IEEE Trans Commun. 50, 1266-1273 (2002). doi:10.1109/TCOMM.2002.801460

5. PK Frenger, N Arne, B Svensson, Decision-directed coherent detection in multicarrier systems on Rayleigh fading channel. IEEE Trans Veh Technol. 48, 490-498 (1999). doi:10.1109/25.752573

6. K Zheng, G Zeng, W Wang, Performance analysis for OFDM-CDMA with joint frequency-time spreading. IEEE Trans Broadcast. 51, 144-148 (2005). doi:10.1109/TBC.2004.839609

7. CB Papadias, B Hochwald, TL Marzetta, A transmitter diversity scheme for wideband CDMA systems based on space-time spreading. IEEE J Sel Areas Commun. 19, 48-60 (2001). doi:10.1109/49.909608

8. L-L Yang, L Hanzo, Performance of broadband multicarrier DS-CDMA using spacetime spreading-assisted transmit diversity. IEEE Trans Wirel Commun. 4. 885-894 (2005)

9. Z Luo, J Liu, M Zhao, M Yuanan Liu, J Gao, Double-orthogonal coded space-time-frequency spreading CDMA scheme. IEEE J Sel Areas Commun. 24(6), 1244-1255 (2006)

10. H Dahman, Y Shayan, X Wang, Space-time-frequency spreading and coding for multi-user MIMO-OFDM systems. in Proc IEEE Int Conf Communications, Beijing, China, 4537-4542 (May 2008)

11. J Wang, X Wang, Optimal linear spacetime spreading for multiuser MIMO communications. IEEE J Sel Areas Commun. 24(1), 113-120 (2006)

12. GG Raleigh, JM Cioffi, Spatio-temporal coding for wireless communication IEEE Trans Commun. 46(3), 357-366 (1998). doi:10.1109/26.662641

13. H Bölcskei, AJ Paulraj, Space-frequency coded broadband OFDM systems. in Proc IEEE Wireless Commun Network Conf, 1-6 (2000)

14. D Tse, $P$ Viswanath, $L$ Zheng, Diversity multiplexing tradeoff in multipleaccess channels. IEEE Trans Inf Theory. 50, 1859-1874 (2004). doi:10.1109/ TIT.2004.833347

15. DGH Bölcskei, AJ Paulraj, On the capacity of OFDM-based spatial multiplexing systems. IEEE Trans Commun. 50(2), 225-234 (2002)

16. Z Liu, Y Xin, GB Giannakis, Space-time-frequency coded OFDM over frequency-selective fading channels. IEEE Trans Signal Process. 50(10), 2465-2476 (2002). doi:10.1109/TSP.2002.803332

17. D Tse, P Viswanath, Fundamentals of Wireless Communication (Cambridge University Press, New York, NY, 2005)

18. L Hanzo, L-L Yang, E-L Kuan, K Yen, Single- and Multi-Carrier DS-CDMA. Multi-User Detection, Space-Time Spreading, Synchronization, Standards and Networking (IEEE Press/Wiley, New York, 2003)

19. S Verdú, Multiuser Detection (Cambridge University Press, Cambridge, UK, 1998)

20. M Jankiraman, Space-Time Codes and MIMO Systems (Artech House, 2004)

21. M Chiani, D Dardan, Improved exponential bounds and approximation for the Q-function with application to average error probability computation. in Proc IEEE Global Telecommunications Conference (GLOBECOM'02), Taipei, Taiwan, 1399-1402 (November 2002)

doi:10.1186/1687-6180-2011-139

Cite this article as: Dahman and Shayan: Performance evaluation of space-time-frequency spreading for MIMO OFDM-CDMA systems. EURASIP Journal on Advances in Signal Processing 2011 2011:139.

\section{Submit your manuscript to a SpringerOpen ${ }^{\circ}$ journal and benefit from:}

- Convenient online submission

- Rigorous peer review

- Immediate publication on acceptance

- Open access: articles freely available online

- High visibility within the field

- Retaining the copyright to your article

Submit your next manuscript at $\gg$ springeropen.com 